\title{
Are there basic laws of quantum information processing?
}

\author{
Michał Horodeckit \\ Department of Mathematics and Physics \\ University of Gdańsk, 80-952 Gdańsk, Poland \\ Ryszard Horodecki块 \\ Institute of Theoretical Physics and Astrophysics \\ University of Gdańsk, 80-952 Gdańsk, Poland
}

\begin{abstract}
We prove within the standard quantum formalism without reduction postulate that the no-cloning theorem and the principle of no-increasing of entanglement under local actions and one-way classical communication are equivalent. We argue that the result is a manifestation of more general principles governing quantum information processing analogous to the thermodynamical laws.
\end{abstract}

PACS numbers: 03.65.Bz, 42.50.Dv, 89.70.+c 


\section{INTRODUCTION}

The recent development of quantum information theory shows that the no-cloning theorem [1] can be considered as a basic principle of quantum information processing (QIP). It provides fundamental bounds for capacities of quantum channels. Quite recently Bennett et al. [2] calculating the capacities of simple quantum channels supplemented (or not) by classical ones, used the theorem as a basic tool for providing upper bounds for the capacities. For example, this allowed to show that the so-called quantum erasure channel with probability $\epsilon=1 / 2$ of erasing has the quantum capacity equal to zero i.e. cannot be used for reliable transmission of quantum information.

On the other hand the no-cloning theorem has been also used in the context of entanglement processing concerning sending quantum information by means of teleportation [3]. Recently Bennett et al. [四] introduced a concept of obtaining pure singlets needed for faithful teleportation from mixed states by means of local quantum operations and classical communication (LCC operations). This is called distillation (or purification) protocol. It appears [5] that the no-cloning theorem allows in some cases to obtain the bounds for the maximal asymptotic yield of produced singlets (called distillable entanglement) for a given mixed state. This becomes more clear, in light of a general connection between quantum channel capacities and distillable entanglement of mixed bipartite states [5]. It involves the fact that many properties of a quantum channel (being in general a completely positive trace preserving map) can be conveniently described by properties of mixed state produced by sending part of maximally entangled bipartite state through it [6]. Thus considering entanglement can be helpful in the investigation of channels and vice-versa. This can be illustrated by the fact that it is only the possibility of sending quantum information by means of entangled state and classical communication (i.e. teleportation) which can make

the capacity of a quantum channel supplemented by two way classical channel to be greater than in the case of the quantum channel alone.

Now let us note that the entanglement processing involves another basic principle. It says 
that entanglement cannot increase under local action and classical communication (we will refer to it as to "no-increasing of entanglement") 円This principle, being proved as a theorem for a particular entanglement measure (entanglement of formation) [5], can be treated as a postulate for any good entanglement measure [5, [, 8]. There was also made some attempt to consider it as an analogue of second law of thermodynamics [7].

Now a fundamental question arises. Is there a connection between the two principles: the first concerning the real and the second - "virtual" quantum information 9 due to entanglement? Note that the possible direct connection could be inferred from the recent result of Bužek et al. [10], who used the imperfect quantum copiers [11] locally to produce two entangled states from one input entangled one. Thus imperfect cloning allows for imperfect "broadcasting inseparability". The fact that the entanglement of the joint output state cannot be greater than the initial one can be viewed as a reflection of imperfection of the copying machines. This suggests that this is just impossibility of cloning which is responsible for the fact that one cannot increase entanglement by LCC operations. Consequently, one can ask the converse question: is it that the no-increasing of entanglement principle implies the no-cloning theorem? More generally, one can ask: are there generic principles of quantum information processing?

The aim of this Letter is to give at least partial answers to the above questions. In sec. 2 we prove the equivalence between no-cloning theorem and the principle of no-creating of entanglement by means of local quantum operations and one-way classical communication. In sec. 3 we consider the equivalence theorem in the context of the recent results in quantum information theory. It leads us to the two principles of quantum information processing being

\footnotetext{
${ }^{1}$ By no-increasing of entanglement under LCC operations we mean that if the output ensemble is $\left\{p_{i}, \varrho_{i}\right\}$ at the input state $\varrho$ then $(\mathrm{i}) \sum_{i} p_{i} E\left(\varrho_{i}\right) \leq E(\varrho)$ (i.e. that the average input entanglement cannot exceed the entanglement of input state) [5] where $\mathrm{E}$ is a given entanglement measure.

${ }^{2}$ The term "virtual" information was introduced in Ref. [9].
} 
analogous to the first and second principles of thermodynamics. In the last section we discuss physical consequences of the principles.

\section{THE EQUIVALENCE THEOREM}

In this section we will show that the no-cloning theorem and the principle of no-increasing of entanglement (in a bit weaker formulation than usual ones) are equivalent. We start with the following formulations

1. No-cloning theorem: There does not exist a quantum operation which clones every

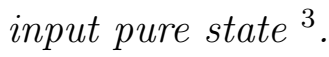

2. No-creating f of entanglement principle: There does not exist an LCC operation which produces singlet pairs from product input state.

In the above formulations we allow the cloning or singlet-creating machines to produce output with any desired but not necessarily perfect accuracy. Note that the principle (2) says simply that the distillable entanglement of product state vanishes which is of course a mathematical fact. Note also that in the above formulation we do not employ any particular entanglement measure. Of course we can divide the LCC operation into the ones involving one- or two-way classical communication. Below we restrict ourselves to the no-one-wayentanglement-creation principle in the form

2'. There does not exist an LCC operation with one-way classical channel which produces singlet pairs from product input state.

\footnotetext{
3 The no-cloning theorem in the strongest version rules out the possibility of cloning even two nonorthogonal states, see Ref. [12

4 According to our formulation of the principle the term "no-creating" seems to be here more appropriate than "no-increasing".
} 
To be precise, by a quantum operation (in short: operation) on a system A we mean a completely positive trace preserving map $\Lambda_{A}: \mathcal{B}\left(\mathcal{H}_{A}\right) \rightarrow \mathcal{B}\left(\mathcal{H}_{A}\right)$ where $\mathcal{H}_{A}$ is the Hilbert space associated with the system $\mathrm{A}$ and $\mathcal{B}\left(\mathcal{H}_{A}\right)$ denotes the set of linear operators acting on $\mathcal{H}_{A}$. Any such a map is of the form

$$
\Lambda_{A}(\varrho)=\sum_{i} V_{i} \varrho V_{i}^{\dagger} \quad \text { with } \sum_{i} V_{i}^{\dagger} V_{i}=I_{A},
$$

where $I_{A}$ denotes identity operator on $\mathcal{H}_{A}$. It is known [13] that the map is also of the form

$$
\Lambda_{A}(\varrho)=\operatorname{Tr}_{C}\left[U \varrho \otimes \omega U^{\dagger}\right]
$$

where $\omega$ is a state on the additional system $\mathrm{C}$ and $\mathrm{U}$ is unitary transformation on the joint system $\mathrm{A}+\mathrm{C}$.

By an LCC operation with one-way classical channel on the compound system $\mathrm{A}+\mathrm{B}$ we mean an operation which can be written in the form

$$
\Lambda_{A B}(\varrho)=\sum_{i} \hat{I}_{A} \otimes \Lambda_{B}^{i}\left(V_{A}^{i} \otimes I_{B} \varrho V_{A}^{i^{\dagger}} \otimes I_{B}\right),
$$

where the $\left\{V_{i}^{A}\right\}$ is a partition of unity on the system $\mathrm{A}, \Lambda_{B}^{i}$ are operations on the system $\mathrm{B}$ and $\hat{I}_{A}$ is identity operation on the system A (i.e. it is a superoperator).

Now we will prove the following

Theorem.- The no-cloning theorem (1) and the principle of no-one-way-creating of entanglement $\left(2^{\prime}\right)$ are equivalent.

Proof.- We will prove that the negations of the principles 1 and $2^{\prime}$ are equivalent. We will usually deal with exact cloning or singlet-creating machines. Then the more general case (where any desired but not exact accuracy is required) will be also true due to compactness of bounded sets in finite-dimensional linear spaces.

Suppose now that given an initial product state $P_{A} \otimes P_{B}$ Alice and Bob are able to produce entangled state $P_{A B}^{e n t}$ by local quantum operation and one-way classical communication i. e. by an operation of the form (3) where $\left\{V_{i}^{A}\right\}$ is the partition of unity corresponding to Alice's generalized measurement and $\Lambda_{B}^{i}$ denotes Bob's operation performed if the i-th outcome is 
obtained by Alice. Consider then an unknown state $P_{C}$ on an additional system $C$ in Alice's laboratory. After performing the operation $\Lambda_{A B}$ the state $P_{C}$ can be teleported to Bob via the state $P_{A B}^{e n t}$. The operation of teleportation is of the same form as $\Lambda_{A B}$

$$
\Lambda_{C A B}^{t e l}(\varrho)=\sum_{i} \hat{I}_{C A} \otimes \tilde{\Lambda}_{B}^{i}\left(\tilde{V}_{C A}^{i} \otimes I_{B} \varrho \tilde{V}_{C A}^{i \dagger} \otimes I_{B}\right)
$$

Combining the two actions on the system $\mathrm{C}+\mathrm{A}+\mathrm{B}$ i.e. $I_{C} \otimes \Lambda_{A B}$ and $\Lambda_{C A B}^{t e l}$ we obtain the joint action

$$
\Lambda_{C A B}(\varrho)=\sum_{i j} \hat{I}_{C A} \otimes \Lambda_{B}^{i j}\left(W_{C A}^{i j} \otimes I_{B} \varrho W_{C A}^{i j \dagger} \otimes I_{B}\right)
$$

where $\Lambda_{B}^{i j}=\tilde{\Lambda}_{B}^{j} \Lambda_{B}^{i}, W_{C A}^{i j}=\tilde{V}_{C A}^{j}\left(I_{C} \otimes V_{A}^{i}\right)$. Then we have for any unknown state $P_{C}$

$$
\operatorname{Tr}_{C A}\left[\sum_{i j}\left(W_{C A}^{i j} P_{C} \otimes P_{A} W_{C A}^{i j \dagger}\right) \otimes \Lambda_{B}^{i j}\left(P_{B}\right)\right]=P_{C}
$$

Now it follows that the operation

$$
\Lambda_{C A B_{1} B_{2} \ldots B_{n}}(\varrho)=\sum_{i j} \hat{I}_{C A} \otimes\left(\otimes_{i=1}^{n} \Lambda_{B}^{i j}\right)\left(W_{C A}^{i j} \otimes I_{B} \varrho W_{C A}^{i j \dagger} \otimes I_{B}\right)
$$

will clone the unknown state $P_{C}$. To see it note that for any operators $A_{i}$ and $B_{i}$ with $\operatorname{Tr} B_{i}=1$ we have

$$
\operatorname{Tr}_{12}\left[\sum_{i} A_{i} \otimes B_{i} \otimes B_{i}\right]=\operatorname{Tr}_{13}\left[\sum_{i} A_{i} \otimes B_{i} \otimes B_{i}\right]=\operatorname{Tr}_{1}\left[\sum_{i} A_{i} \otimes B_{i}\right] .
$$

Then for any state $P_{C}$

$$
\operatorname{Tr}_{C A}\left[\Lambda_{C A B_{1} B_{2} \ldots B_{n}}\left(P_{C} \otimes P_{A} \otimes\left(\otimes_{i=1}^{n} P_{B}\right)\right)\right]=\otimes_{i=1}^{n} P_{C}
$$

Thus from no-cloning theorem follows the no-entanglement-creating one.

Suppose now conversely, that we are able to clone quantum states i.e. that there exists an operation $\Lambda_{A C_{1} \ldots C_{n}}$ and state $\otimes_{i=1}^{n} P_{C}$ such that for any unknown state $P_{A}$

$$
\Lambda_{A C_{1} \ldots C_{n}}^{c o p y}\left(P_{A} \otimes\left(\otimes_{i=1}^{n} P_{C}\right)\right)=\otimes_{i=1}^{n+1} P_{A}
$$

Performing this operation Alice obtains $n+1$ (arbitrarily good) copies of the initial state $P_{A}$. Then there exists a measurement $\left\{P_{i}\right\}$ on the system $A C_{1} \ldots C_{n}$ such that to any outcome 
$i$ Alice can ascribe a guessed state $\tilde{P}_{i}$ with $\sum_{i} p_{i} \tilde{P}_{i}$ closely approximating $P_{A}$ for large n; $p_{i}$ denote the probabilities of the corresponding outcomes (for optimal measurement in the spin- $-\frac{1}{2}$ case see [20]). The two actions of Alice - copying and measurement - can be treated jointly as a generalized measurement performed on the system A given by partition of unity $\left\{V_{A}^{i}\right\}$. Now obtained an outcome $i$ Alice sends it classically to Bob, who subjects his initial state $P_{B}$ to an operation $\Lambda_{B}^{i}$ to obtain $\tilde{P}_{i}$

$$
\Lambda_{B}^{i}\left(P_{B}\right)=\tilde{P}_{i}
$$

Combining actions of Alice and Bob we obtain joint operation $\Lambda_{A B}^{n}$ which is of course LCC operation with one-way classical channel

$$
\Lambda_{A B}^{N}(\varrho)=\sum_{i} I_{A} \otimes \Lambda_{B}^{n i}\left(V_{A}^{n i} \otimes I_{B} \varrho V_{A}^{n i \dagger} \otimes I_{B}\right)
$$

and which has the property that for all $P_{A}$ and for $n$ large enough $\operatorname{Tr}_{A}\left[\Lambda_{A B}^{n}\left(P_{A} \otimes P_{B}\right]\right.$ closely approximates $P_{A}$. Now it suffices to show that entanglement can be created by means of a map $\Lambda_{A B}$ for which there holds exact equality

$$
\operatorname{Tr}_{A}\left[\Lambda_{A B}\left(P_{A} \otimes P_{B}\right]=P_{A}\right.
$$

To create entanglement Alice prepares entangled state $P_{A C}^{e n t}$ on the system $A+C$, where $C$ is ancilla, and then Alice and Bob apply the operation $\Lambda_{A B}$. Now we have to show that if the latter map flips any state $P_{A}$ onto Bob's system, then also the entanglement will be suitably swapped. Equivalently one can show that $\Lambda_{A B}$ followed by the flip operator $\left(\Lambda_{V}(P \otimes Q)=Q \otimes P\right)$ will leave entanglement $\mathrm{A}$ and $\mathrm{C}$ undisturbed. Consider then the map $\Lambda_{A}(\varrho)=\operatorname{Tr}\left(\Lambda_{V} \Lambda_{A B}\left(\varrho \otimes P_{B}\right)\right)$. By the property (13) such a map must be identity, so acting on half of entangled state on the system $\mathrm{A}+\mathrm{C}$ will leave it undisturbed

$$
\left(\Lambda_{A} \otimes I_{C}\right)\left(P_{A C}^{e n t}\right)=P_{A C}^{e n t}
$$




\section{POSSIBLE IMPLICATIONS OF THE EQUIVALENCE THEOREM: PRINCIPLES OF QUANTUM INFORMATION PROCESSING}

Now it is natural to ask what are physical implications of the equivalence theorem. To give answer to this question, we adopt the view according to which quantum information is

a physical quantity [21,22 similarly as energy in thermodynamics. Below we will consider the physical meaning of the equivalence theorem in this spirit.

A. First principle. In the proof of the theorem we have utilized the fact that if a physical operation sends any state undisturbed then it will also swap entanglement without destroying it. To understand it, note that if we work within the orthodox quantum formalism (without the von Neumann projection postulate) then acting on one part of entangled system, we have no way to annihilate the entanglement. The latter can change only by means of interacting of the both entangled subsystems. The only way we can disturb it is to entangle the considered subsystem with another one. Then the entanglement will not of course vanish but it will spread over all the three subsystems. Now if the action leaves any state of the subsystem undisturbed, then it obviously does not entangle it with any other one. Consequently, the entanglement of the initial system remains unchanged. The same concerns the operation which sends any state without any disturbance to another system. Then it will also perfectly swap entanglement.

Note that the present reasoning can be viewed as utilizing of some implication of conservation of quantum information (which can be called first principle of QIP in analogy to that of thermodynamics). It can be formulated as follows

$I^{\prime}$. The entanglement of the compound system does not change under unitary processes on one of the subsystems.

Indeed the equivalence theorem strongly confirms the recently more and more common view that entanglement is simply a form of quantum information. Then the rules of processing of quantum information can always be formulated in two ways. The above formulation expresses the principle of conservation of information in the language of virtual quantum 
information represented by half of entangled state. We can express it also in terms of real quantum information, i.e. the one associated with unknown parameters of state of a particle. Consider for this purpose a system in an unknown state representing a qubit of quantum information and another system in an established state (hence representing no quantum information). Now, if the unitary transformation over the whole system is performed, one can observe how the information is spread over the system. As there was one qubit of initial amount of information and the information of the whole system cannot change, after evolution we are left also with one qubit of information. However, the latter is in general no longer contained in the first subsystem, but part of it could be flipped onto the second one or changed into quantum entanglement of the whole system. Then the alternative formulation of the first principle of QIP can be as follows

I. For a compound quantum system the sum of information contained in the subsystems and the information contained in entanglement is conserved in unitary processes.

As an illustration of the above principle can serve the observation [15, 16] that during quantum copying process the information is not lost but can be found in entanglement of the output qubits and copying machine. Note that here the entanglement represents real quantum information, as it contains the unknown parameters of the superposition of the input qubit, while the virtual information is identified with entanglement itself or, more precisely, half of a system being in a known, established entangled state.

B. Second principle. Having formulated the information conservation principle in the two ways we are in position to interpret the result of the previous section. The two equivalent statements 1 and $2^{\prime}$ are formulations of the same restriction for quantum information processing in terms of quantum real and virtual information respectively. Following the proof, one can see that the no-cloning theorem says in fact that we cannot send quantum real information through classical channel. As we work within quantum formalism (where there are no "classical" channels) the above means that it is not possible to flip a qubit from one system to another by some sort of quantum operations on the whole system i.e. by the operations of the form (3), where we think about them as if they were a part of unitary 
transformation over the system of interest plus some unobserved ancilla. Then the equivalence theorem says that this impossibility is equivalent to impossibility of swapping half of entangled system onto another one by means of such operations. The latter impossibility we have called in short no-creating entanglement principle. However it must be emphasized here, that it is not a consequence of information conservation. The latter implies merely that acting on one of the system we cannot create or annihilate entanglement. Now the map (3) acts on both the subsystems. Thus the equivalent no-cloning or no-creation of entanglement principles constitute a different physical law which says that neither real nor virtual quantum information can be transmitted by such type of channels. One can consider stronger prohibition: quantum information cannot be transmitted by a so-called separable superoperator 8, 17]

$$
\Lambda(\varrho)=\sum_{i} V_{A}^{i} \otimes V_{B}^{i} \varrho V_{A}^{i \dagger} \otimes V_{B}^{i \dagger}
$$

Under the consideration one can formulate the second principle of QIP as follows

II. One cannot spread quantum real information from an initial system onto larger number of systems without changing part of it into entanglement.

To formulate it in terms of virtual information, imagine that two systems $\mathrm{A}$ and $\mathrm{B}$ are entangled, and we can act only on the system A and some additional system $\mathrm{C}$. Then it is impossible to make $\mathrm{C}$ entangled with $\mathrm{B}$ in such a way that all entanglement of the system $\mathrm{ABC}$ is contained in the subsystems $\mathrm{AB}$ and $\mathrm{AC}$. This can be formulated almost identically as in the case of real information, if we recall the idea of "entangled entanglement" 18. Indeed, similarly as the quantum real information is partly converted into entanglement, the lacking part of virtual information which is not contained in the pairs $\mathrm{AB}$ and $\mathrm{AC}$, but only in the whole system ABC, may be thought as being converted into "entanglement of entanglement". In this way we have come to the suitable formulation the second principle of QIP in terms of virtual information

II'. It is impossible to spread virtual information without changing part of it into entangled entanglement. 
It can be seen that the second principle of QIP implies the no-cloning principle. As the latter has been proved to be equivalent to no-creation of entanglement, we see that our formulations of the second principle are closely related to the one proposed by Popescu and Rohrlich [7] who postulated no-increasing of entanglement as an analogue to the thermodynamical law.

\section{DISCUSSION AND CONCLUSION}

That the second principle of QIP may play the role analogous to the second law of thermodynamics we can see from the fact that in our formulation the former implies the nocloning theorem. As it was mentioned, the latter provides fundamental limits for capacities

of quantum channels. Analogously, the impossibility of sending information by a channel constituted by local quantum operations and two-way classical communication (also implied by the second principle) provides bounds for capacities of quantum channels supplemented with two-way classical channels. This is analogous to the fact that it is just the second law of thermodynamics what determines the limits for efficiency of cyclic machines acting between two heat reservoirs. Now the parameters of noisy channels can be considered as a counterpart of the temperatures $T_{1}$ and $T_{2}$ of reservoirs, while the error-correcting protocols would correspond to the thermodynamical processes. The central problem in the quantum information theory of finding the optimal protocol for a given noisy channel can be then compared with the problem of finding the engine (i.e. the cyclic process) with the best efficiency for given temperatures $T_{1}$ and $T_{2}$, what was done by Carnot.

Let us now consider briefly the problem of the "time arrow" in the context of the second law of QIP. It may be objected that the latter cannot be suitable counterpart of the thermodynamical principle as there is no irreversibility in the used formalism. However, the discussed notions as e.g. quantum information, can be considered independently of quantum formalism. For example, if the quantum computer were realized in laboratories, the quantum information would not be an abstract notion any more, but it would become a very concrete 
physical quantity. Then we can observe an analogue of the "arrow" which is permanently ascribed to the second law of thermodynamics. Namely in the real world we still observe the decoherence [19]. The latter is nothing else but in practice irreversible entangling of a number of systems. Thus, although the decoherence is, in standard quantum formalism, logically reversible, in real world there is a tendency that quantum information prefers to be spread over many systems, and necessarily changes into uncontrolled entanglement. But what is uncontrolled entanglement? According to our analogy, it is simply "information heat flaw". Indeed, the thermodynamical heat flaw means in fact increase of internal energy of uncontrolled reservoir.

Here a question arises: where is the place of the quantum entropy in the above framework? This is a fundamental problem, as the second law of thermodynamics is inherently connected with the notion of entropy. But in our approach the entropy of the universe is constant, as we consider the latter to be a closed system, to keep the conservation information principle valid f A possible way out is that according to the interpretation of our formulation of second law of QIP, one can divide the universe into the elementary subsystems and note that the tendency of increasing of entanglement between them makes their entropies to become larger and larger. Then, in result, the sum of partial entropies increases. It can be viewed as a suitable analogue of the statement in the standard thermodynamics that "the entropy of the world increases".

It should be emphasized here that the quantum information has no its own dynamics, but it is carried by the particles the motion of which is governed by a dynamics which is unavoidably connected with the concept of energy. In fact there are two levels in nature: energetic (thermodynamical) and informational (logical). They are mutually coupled because the dynamics starts with some initial conditions which can be considered an initial

\footnotetext{
${ }^{5}$ This is consistent with the concept of unitary information field [21], which rests on the assumption that the notion of information is a fundamental category in the description of reality.
} 
package of information. Then it follows that the informational "arrow" is a reflection of the thermodynamical one. This suggests, that the developed here analogy between quantum informational and thermodynamical processes is not just a formal one, but has a deeper physical justification.

Finally we have to discuss our results in the context of the postulate reduction of the wave function. In the mathematical proof and the discussion in the previous section we have considered Alice's and Bob's actions to be unitary transformations over large system. This cannot be so, if we treat reduction of wave packet as a real physical process. Then the whole Alice-Bob action cannot be thought as a part of unitary transformation and then the proof of equivalence is not valid any more. Such a situation arises when the observers, Alice and Bob, are not considered to be a part of the quantum world but rather belong to another "classical" world [23]. Then indeed, the wave packet reduction actually holds, the quantum information is lost (entanglement is annihilated) and we must abandon the information conservation principle. One could ask now, when the joint action of Alice and Bob can be viewed as a part of unitary transformation? The answer is: only if we include the observers into the quantum world. Then entanglement cannot be annihilated by any action which does not affect both entangled systems. In other words, to keep the quantum information conservation valid, one is forced to work within the interpretations of quantum mechanics which dispense with the postulate of collapse of wave function 9 . This means that the validity of the first principle and the equivalence theorem appears to be interpretationdependent.

To summarize, we have proved that within the quantum formalism without reduction postulate the no-cloning theorem is equivalent to the fact that one cannot produce entanglement from product state by means of local quantum operations and one-way classical communication. Then we have postulated some principles of QIP analogous to those in

\footnotetext{
${ }^{6}$ See in this context Refs. 24,25].
} 
thermodynamics. We have expressed both the principles by means of real and virtual information where the real quantum information is represented by an unknown quantum state while virtual one - by half of entangled system. The statements proved to be equivalent appear now to constitute the same restriction for quantum information processing expressed in the two different ways. They appear to be a consequence of the second principle of QIP. The first principle, being information conservation law, does not hold if the postulate of reduction of wave packet is considered as real physical process.

The authors are grateful to Charles Bennett, Paweł Horodecki, Martin Plenio and Vlatko Vedral for stimulating discussions and helpful comments. This work is supported in part by Polish Committee for Scientific Research, Contract No. 2 P03B 02412. 


\section{REFERENCES}

* E-mail address: michalh@iftia.univ.gda.pl

** E-mail address: fizrh@univ.gda.pl

[1] W. K. Wooters and W. H. Zurek, Nature (London) 299802 (1982); see also generalization of the no-cloning theorem for mixed states in H. Barnum, C. M. Caves, C. A. Fuchs, R. Jozsa and B. Schumacher, Phys. Rev. Lett. 76, 2818 (1996).

[2] C. H. Bennett, D. Di Vincenzo and J. Smolin, "Capacities of Quantum Erasure Channels", Report No. quant-ph/9701015.

[3] C. Bennett, G. Brassard, C. Crepeau, R. Jozsa, A. Peres and W. K. Wootters, Phys. Rev. Lett. 70, 1895 (1993).

[4] C. H. Bennett, G. Brassard, S. Popescu, B. Schumacher, J. Smolin and W. K. Wootters, Phys. Rev. Lett. 76, 722 (1996).

[5] C. H. Bennett, D. P. Di Vincenzo, J. Smolin and W. K. Wootters, Phys. Rev. A 54, 3814 (1997).

[6] B. Schumacher and M. A. Nielsen, Phys. Rev. A 54, 2629 (1996).

[7] S. Popescu and D. Rohrlich "On the Measure of Entanglement for Pure States" Report No. quant-ph/9610044.

[8] V. Vedral, M. B. Plenio, M. A. Rippin and P. L. Knight, Phys. Rev. Lett. 78 (1997) 2275

[9] N. J. Cerf and C. Adami, "Negative Entropy and Information in Quantum Mechanics", Report No. quant-ph/9512022.

[10] V. Bužek, V. Vedral, M. B. Plenio, P. L. Knight, and M. Hillery, Phys. Rev. A 55 (1997) 3327. 
[11] V. Bužek and M. Hillery, Phys. Rev. A 54, 1844 (1996).

[12] H. P. Yuen, Phys. Lett A 113, 405 (1986).

[13] K. Kraus, States, Effects and Operations: Fundamental Notions of Quantum Theory, Wiley, New York, 1991.

[14] G. M. D’Adriano and H. P. Yuen, Phys. Rev. Lett. 762832 (1996).

[15] V. Bužek, S. L. Braunstein, M. Hillery and D. Bruß, "Quantum copying: A network", Report No. quant-ph/9703046.

[16] N. Gisin and S. Massar, "Optimal Quantum Cloning Machines", Report No. quantph/9705046.

[17] M. Rains, "Entanglement of purification via separable superoperators", Report No. quant-ph/9707002.

[18] G. Krenn and A. Zeilinger, Phys. Rev. A 541793 (1996).

[19] W. H. Zurek, Physics Today, October 36 (1991).

[20] S. Massar and S. Popescu, Phys. Rev. Lett. 74, 1259 (1995).

[21] R. Horodecki, Ann. Phys. (Leipzig) 48 479, (1991).

[22] R. Landauer Phys. Lett. A 217188 (1996).

[23] Ph. Blanchard and A. Jadczyk, Phys. Lett. A 175 157, (1993).

[24] H. Everett, Rev. Mod. Phys. 29454 (1957).

[25] D. Bohm and B. J. Hiley, Phys. Rep. 144, 323 (1987). 